\title{
How Slowly Can Quadrature Formulas Converge?
}

\author{
By Peter R. Lipow and Frank Stenger
}

\begin{abstract}
Let $\left\{Q_{n}\right\}_{n=1}^{\infty}$ denote a sequence of quadrature formulas, $Q_{n}(f) \equiv \sum_{j=1}^{k_{n}} w_{j}^{(n)} f\left(x_{j}^{(n)}\right)$, such that $Q_{n}(f) \rightarrow \int_{0}^{1} f(x) d x$ for all $f \in C[0,1]$. Let $0<\epsilon<\frac{1}{4}$ and a sequence $\left\{a_{n}\right\}_{n=1}^{\infty}$ be given, where $a_{1} \geqq a_{2} \geqq a_{3} \geqq \cdots$, and where $a_{n} \rightarrow 0$ as $n \rightarrow \infty$. Then there exists a function $f \in C[0,1]$ and a sequence $\left\{n_{k}\right\}_{k=1}^{\infty}$ such that $|f(x)| \leqq 2 a_{1} /|(1-4 \epsilon)|$, and such that $\int_{0}^{1} f(x) d x-Q_{n_{k}}(f)=a_{k}, k=1,2,3, \cdots$.
\end{abstract}

1. Introduction and Statement of Results. We consider a sequence of quadrature formulas $\left\{Q_{n}\right\}_{n=1}^{\infty}$ defined by

$$
Q_{n} f=\sum_{i=1}^{k_{n}} w_{i}^{(n)} f\left(x_{i}^{(n)}\right)
$$

where $\left\{k_{n}\right\}_{n=1}^{\infty}$ is a sequence of (increasing) positive integers and $0 \leqq x_{i}^{(n)} \leqq 1$ for all $n$ and $j$. The quadrature formulas, we assume, are such that

$$
\lim _{n \rightarrow \infty} Q_{n} f=I f \equiv \int_{0}^{1} f(x) d x
$$

for all functions $f$ that are continuous on the closed interval $[0,1]$; that is, for all $f$ in $C[0,1]$. For example, the Gaussian quadrature formulas and the well-known trapezoidal formulas have these properties.

In this paper, we show that no matter what the sequence $\left\{Q_{n}\right\}_{n=1}^{\infty}$ defined by (1.1) and (1.2) is, there is a function $f$ in $C[0,1]$ for which $\left\{Q_{n} f\right\}_{n=1}^{\infty}$ converges to If very slowly. That is, the assumption of continuity is not enough to insure the rapid convergence of any quadrature scheme. More precisely, our main result is the following:

THEOREM 1. Let a sequence of quadrature formulas $\left\{Q_{n}\right\}_{n=1}^{\infty}$ defined by (1.1) and satisfying (1.2) for all $f$ in $C[0,1]$ be given, and let $\left\{a_{n}\right\}$ be any sequence of numbers such that

$$
\lim _{n \rightarrow \infty} a_{n}=0,
$$

and

$$
S \equiv \sum_{n=1}^{\infty}\left|a_{n}-a_{n+1}\right|<\infty
$$

Received February 16, 1972.

AMS 1970 subject classifications. Primary 65D30.

Key words and phrases. Quadrature rules, convergence.

Copyright @ 1972, American Mathematical Society 
Corresponding to any $\epsilon$ such that $0<\epsilon<\frac{1}{4}$, there exists a function $f$ that is continuous and bounded by $2 S /(1-4 \epsilon)$ on the interval $[0,1]$, and an increasing sequence of positive integers $\left\{n_{k}\right\}_{k=1}^{\infty}$ such that

$$
\text { If }-Q_{n_{k}} f=a_{k} \quad \text { for } k=1,2, \cdots .
$$

We also have

COROllaRy 2. For every integer $N>0$, there exists a polynomial $P$ bounded on the interval $[0,1]$ by $2 S /(1-4 \epsilon)$ such that

$$
I P-Q_{n k} P=a_{k} \quad \text { for } k=1,2, \cdots, N .
$$

Remark 3. We remark that if the sequence $\left\{a_{k}\right\}_{k=1}^{\infty}$ is a monotonically increasing or decreasing sequence of real numbers, then $S=\left|a_{1}\right|$.

In the following section, we will construct the function $f$ of Theorem 1 as a uniformly convergent sum of linear spline functions (broken linear functions) on the interval $[0,1]$. This constructive proof of Theorem 1 leads to an elementary proof of Corollary 2. In the final section, we consider a particular sequence of quadrature formulas and show the easier calculation involved in this case.

Some historical remarks are in order. Let $P_{n}$ denote the set of polynomials of degree $n-1$ in $x, n=1,2,3, \cdots$. In 1938, Bernstein [1] proved that, given any sequence of positive numbers $a_{1} \geqq a_{2} \geqq a_{3} \geqq \cdots \geqq a_{n} \geqq \cdots \geqq 0$, where $a_{n} \rightarrow 0$ as $n \rightarrow \infty$, there exists a function $f \in C[0,1]$ such that

$$
\inf _{p \in P_{n}}\left\{\sup _{x \in(0,1)}|f(x)-p(x)|\right\}=a_{n}, \quad n=1,2,3, \cdots .
$$

This result has since been cast in the terminology of best approximation in normed linear spaces (Timan [2, p. 40]). From the point of view of practical applications, Bernstein's theorem tells us that there are continuous functions defined on the interval $[0,1]$ which cannot be approximated to a desired accuracy by polynomials.

Our paper extends a recent result of Chui [5] who proved that given $\left\{a_{n}\right\}_{n=1}^{\infty}$, a sequence of positive numbers which converges monotonically to zero, there exists a Riemann integrable function $f$ such that

$$
\left|\int_{0}^{1} f(x) d x-\frac{1}{n} \sum_{k=1}^{n} f\left(\frac{k}{n}\right)\right| \geqq a_{n} \text { for } n=1,2, \cdots \text {. }
$$

In 1933 Pólya [3] constructed an analytic function for which the Newton-Cotes quadrature scheme diverges. Pólya's result was preceded by an interesting asymptotic estimate of the remainder of Newton-Cotes quadrature due to Ouspensky [6]; in [6], Ouspensky concluded that the Newton-Cotes formulas were devoid of any practical value. Thus, while proofs of convergence of quadrature schemes applied to continuous functions are very interesting (see Pólya [3] and Espinoza-Maldonado and Byrne [4]), the point of our paper in the spirit of Ouspensky is that when quadrature formulas are applied to continuous functions, the results may be devoid of any practical value.

2. Constructive Proofs. Let $\Pi_{i}$ denote the set of evaluation points of $Q_{n_{i}}$. The function $f$ will be of the form 


$$
f(x)=\sum_{i=1}^{\infty} \alpha_{i} s_{i}(x)
$$

where the $s_{i}$ are to be constructed and the $\alpha_{i}$ are to be determined.

Choice of $n_{i}$ and Construction of $s_{i}(x)$. We fix $\epsilon$ such that $0<\epsilon<\frac{1}{4}$. Then, by (1.2), there is an integer $n_{1}$ such that

$$
\left|Q_{n_{1}} 1-I(1)\right|<\epsilon / 2 .
$$

We now define $s_{1}$ to be the linear spline function whose graph has the vertices

(i) $s_{1}(x)=1$ for all $x \in \Pi_{1}$, and

(ii) $s_{1}(x)=0$ for all $x$ midway between consecutive points of $\Pi_{1}$, and also for $x \in\{0,1\}-\Pi_{1}$.

Thus, if $\Pi_{1}=\left\{z_{1}, z_{2}, z_{3}\right\}$, we would have the following graph:

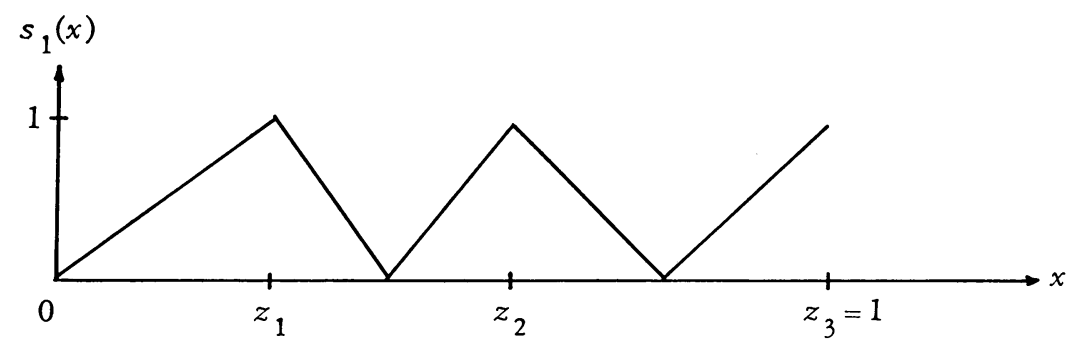

Since $s_{1}(x)=1$ on $\Pi_{1}$, we have, by (2.2),

$$
Q_{n_{1}} s_{1}=1+\eta_{11} \quad \text { where }\left|\eta_{11}\right|<\epsilon / 2 .
$$

Also, clearly,

$$
\int_{0}^{1} s_{1}(x) d x=\frac{1}{2}
$$

We now proceed to the $p$ th stage and suppose we have picked $n_{1}, n_{2}, \cdots, n_{p-1}$ so that

$$
\int_{0}^{1} s_{i}(x) d x=\frac{1}{2} \quad \text { for } i=1,2, \cdots, p-1
$$

Let $\delta_{p}=\epsilon / p 2^{p}$. We cover the union of the $\Pi_{i}(i=1,2, \cdots, p-1)$ with a finite union of open (and, possibly, half-open) intervals of total length $\delta_{p} / 3$. We call this union $C_{p}$ and cover its closure with another union of open (and half-open) intervals, $B_{p}$, this time of total length $2 \delta_{p} / 3$.

We now define a preliminary function $T_{p}$ to be continuous on the interval $[0,1]$ and such that

(i) $T_{p}(x)=0$ for $x$ in $C_{p}$,

(ii) $T_{p}(x)=1$ for $x$ in $[0,1]-B_{p}$, and

(iii) $T_{p}(x)$ is linear elsewhere.

Thus, we might have the graph 


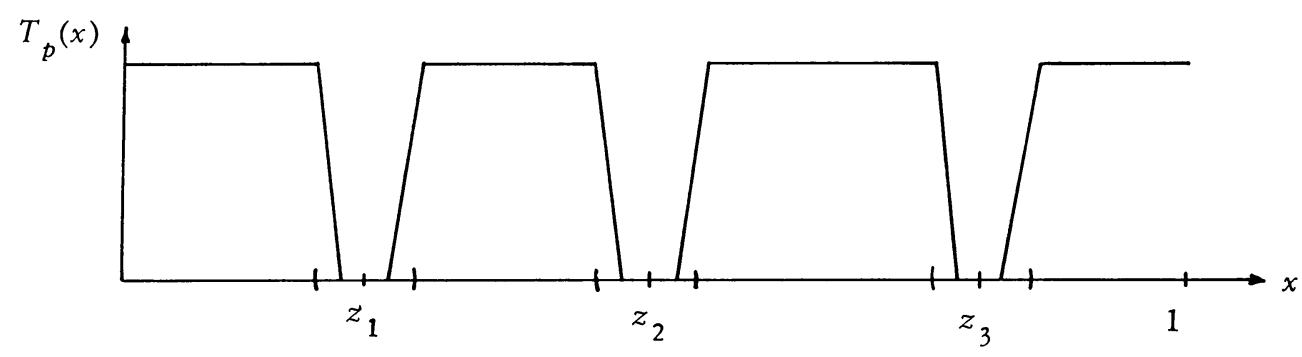

where the union of the $\Pi_{i}$ is $\left\{z_{1}, z_{2}, z_{3}\right\}$. Then, from the definition of $T_{p}$, we clearly have

$$
\int_{0}^{1}\left(1-T_{p}(x)\right) d x \leqq 2 \delta_{p} / 3 .
$$

By (1.2), (2.5) and (2.6) there is an integer $n_{p}$ such that

$$
\begin{array}{ll}
Q_{n_{p}} s_{j}=\frac{1}{2}+\eta_{p i} & \text { for } j=1,2, \cdots, p-1 \text { where }\left|\eta_{p i}\right|<\delta_{p}, \quad \text { and } \\
Q_{n_{p}} T_{p}=1+\eta_{p p} & \text { where }\left|\eta_{p p}\right|<\delta_{p} .
\end{array}
$$

Finally, we define $s_{p}(x)$ to be the linear spline function which is identical to $T_{p}(x)$ on the closure of the set $B_{p}-C_{p}$ and whose graph has the following additional vertices:

(i) $s_{p}(x)=1$ for $x \in \Pi_{p} \cap\left([0,1]-B_{p}\right)$,

(ii) $s_{p}(x)=0$ for $x \in \bigcup_{i=1}^{p-1} \Pi_{i}, x \in\{0,1\}-\Pi_{p}$ and $x \in \Pi_{p} \cap C_{p}$,

(iii) $s_{p}(x)=0$ for all $x$ halfway between two consecutive "one" vertices so far defined, and, in (ii).

(iv) $s_{p}(x)=1$ for all $x$ halfway between two consecutive "zero" vertices defined

Then, $s_{p}(x)$ has the following properties:

$$
\begin{gathered}
Q_{n_{p}} S_{p}=Q_{n_{p}} T_{p} \quad \text { since } s_{p}=T_{p} \text { on } \Pi_{p} . \\
\int_{0}^{1} s_{p}(x) d x=\frac{1}{2} .
\end{gathered}
$$

In this manner, we pick the subsequence $\left\{n_{i}\right\}_{i=1}^{\infty}$ and the functions $\left\{s_{i}\right\}_{i=1}^{\infty}$.

Determination of $\alpha_{i}$. We can now apply the linear functional $I-Q_{n_{p}}$ to the function $f$ of (2.1), and, at the same time, impose the condition (1.5). We then obtain, using (2.6)-(2.10), the following infinite system of linear equations:

$$
\frac{1}{2} \sum_{j=1}^{\infty} \alpha_{i}-\left(\sum_{i=1}^{p-1} \alpha_{j}\left(\frac{1}{2}+\eta_{p i}\right)+\alpha_{p}\left(1+\eta_{p p}\right)\right)=a_{p} \text { for } p=1,2, \cdots .
$$

If we subtract two such consecutive equations, we obtain the recurrence relation

$$
\begin{aligned}
\alpha_{p+1}\left(1+\eta_{p+1, p+1}\right)= & a_{p}-a_{p+1}+\alpha_{p}\left(\frac{1}{2}+\eta_{p p}-\eta_{p+1, p}\right) \\
& +\sum_{j=1}^{p-1} \alpha_{j}\left(\eta_{p j}-\eta_{p+1, i}\right) .
\end{aligned}
$$


Setting $\alpha_{1}=0$, we can use (2.12) to solve for all the $\alpha_{i}$.

It should be noted here that the passage from (2.11) to (2.12) is so far only a formal one, since the infinite series on the left-hand side of (2.11) has not yet been shown to converge. This will be shown in the next part of the proof.

$f$ is Continuous. In order to complete the proof of Theorem 1 we need only show that the $\alpha_{i}$, as determined above, make the function $f$ of (2.1) continuous on the interval $[0,1]$. Thus, we must show that the infinite series $(2.1)$ converges uniformly on the interval $[0,1]$. Since $\left|s_{i}(x)\right| \leqq 1$ for all $x$ and for every integer $i$, we need only show that

$$
\sum_{p=1}^{\infty}\left|\alpha_{p}\right|<\infty .
$$

Indeed, from (2.12) and the bounds on $\eta_{p i}$ given in (2.7),

$$
\left|\alpha_{p+1}\right| \leqq\left|a_{p}-a_{p+1}\right|+\frac{1}{2}\left|\alpha_{p}\right|+\frac{\epsilon}{2^{p-1}} \sum_{j=1}^{p+1}\left|\alpha_{j}\right|
$$

We now sum each side of (2.14) from 1 to $N$, where $N$ is an arbitrary but fixed positive integer. Replacing the sum $\sum_{i=1}^{p+1}\left|\alpha_{j}\right|$ on the right-hand side by $\sum_{i=1}^{N+1}\left|\alpha_{j}\right|$ and adding $\frac{1}{2}\left|\alpha_{N+1}\right|$ to the right-hand side, we obtain

$$
\sum_{p=1}^{N}\left|\alpha_{p+1}\right| \leqq \sum_{p=1}^{N}\left|a_{p}-a_{p+1}\right|+\frac{1}{2} \sum_{p=1}^{N+1}\left|\alpha_{p}\right|+\epsilon \sum_{p=1}^{N} \frac{1}{2^{p-1}} \sum_{j=1}^{N+1}\left|\alpha_{j}\right| .
$$

Transposing and simplifying gives

$$
\sum_{p=1}^{N}\left|\alpha_{p+1}\right|\left\{1-\frac{1}{2}-2 \epsilon\left(1-\frac{1}{2^{N}}\right)\right\} \leqq \sum_{p=1}^{\infty}\left|a_{p}-a_{p+1}\right|<\infty \quad \text { by (1.4). }
$$

Now letting $N \rightarrow \infty$ on the left, we obtain

$$
\sum_{p=1}^{\infty}\left|\alpha_{p+1}\right| \leqq \frac{2}{1-4 \epsilon} \sum_{p=1}^{\infty}\left|a_{p+1}-a_{p}\right|
$$

This completes the proof of Theorem 1 .

Proof of Corollary 2. The proof of Corollary 2 is similar to that of Theorem 1, with one exception. Here we also need to use Weierstrass' approximation theorem, which enables us to approximate each continuous function $s_{p}$ that we constructed in the proof of Theorem 1 by a polynomial $q_{p}$, such that $\max _{x \in[0,1]}\left|s_{p}(x)-q_{p}(x)\right|<$ $\delta_{p} / 2$ for $p=1,2, \cdots, N$. We omit the details of this proof.

3. A Special Case. We consider the following sequence of midpoint quadrature formulas which certainly satisfy (1.2):

$$
Q_{n} f=\frac{1}{2^{n-1}}\left[f\left(\frac{1}{2^{n}}\right)+f\left(\frac{3}{2^{n}}\right)+f\left(\frac{5}{2^{n}}\right)+\cdots+f\left(\frac{2^{n}-1}{2^{n}}\right)\right] .
$$

In this case there are no repeating evaluation points, and we can therefore choose $n_{i}=i$, and define $s_{i}$ to be the linear spline whose graph has the vertices

(i) $s_{i}(x)=1$ for $x \in \Pi_{i}$, and

(ii) $s_{i}(x)=0$ for $x \in \bigcup_{j=1}^{i-1} \Pi_{i}$ and also for $x=0,1$. 
Then, as in (2.9),

$$
\int_{0}^{1} s_{i}(x) d x=\frac{1}{2} \quad \text { for all } i
$$

Instead of (2.6) and (2.7), we have the exact relations

$$
\begin{array}{rlr}
Q_{i} s_{j}=\frac{1}{2} & \text { if } j<i, \\
=1 & \text { if } j=i, \\
=0 & \text { if } j>i .
\end{array}
$$

Instead of (2.11), we have

$$
\frac{1}{2} \sum_{i=1}^{\infty} \alpha_{i}-\frac{1}{2} \sum_{i=1}^{p-1} \alpha_{i}-\alpha_{p}=a_{p} \text { for } p=1,2, \cdots .
$$

Subtracting two such consecutive equations gives the recurrence relation

$$
\alpha_{p+1}=a_{p}-a_{p+1}+\frac{1}{2} \alpha_{p},
$$

which can be solved explicitly in terms of $\alpha_{1}$ to give

$$
\begin{aligned}
\alpha_{p+1}= & a_{p}-a_{p+1}+\frac{1}{2}\left(a_{p-1}-a_{p}\right) \\
& +\frac{1}{2^{2}}\left(a_{p-2}-a_{p-1}\right)+\cdots+\frac{1}{2^{p-1}}\left(a_{1}-a_{2}\right)+\frac{1}{2^{p}} \alpha_{1} .
\end{aligned}
$$

The function $f$ of the form (2.1) is easily seen to be continuous this time, since

$$
\sum_{p=1}^{\infty}\left|\alpha_{p}\right| \leqq 2 \sum_{p=1}^{\infty}\left|a_{p}-a_{p+1}\right|+2\left|\alpha_{1}\right| .
$$

If we take $\alpha_{1}=0$, we note that

$$
|f(x)| \leqq 2 \sum_{p=1}^{\infty}\left|a_{p+1}-a_{p}\right| .
$$

Centre de Recherches Mathématiques

Université de Montréal

Montréal, Québec, Canada

1. S. N. BeRNSTEIN, "On the inverse problem of the theory of best approximation of continuous functions," Sochineniya, v. 2, 1938, pp. 292-294. (Russian)

2. A. F. Timan, Theory of Approximation of Functions of a Real Variable, Fizmatgiz, Moscow, 1960; English transl., Internat. Series of Monographs in Pure and Appl. Math., vol. 34, Macmillan, New York, 1963. MR 22 \#8257; MR 33 \#465.

3. G. PóLYA, "Über die Konvergenz von Quadraturverfahren," Math. Z., v. 37, 1933, pp. 264-286.

4. R. EsPinOza-MaldonAdo \& G. D. BYRNE, "On the convergence of quadrature formulas," SIAM J. Numer. Anal., v. 8, 1971, pp. 110-114.

5. C. K. CHUI, "Concerning rates of convergence of Riemann sums," J. Approximation Theory, v. 4, 1971, pp. 279-287.

6. J. Ouspensky, "Sur les valeurs asymptotiques des coefficients de Cotes," Bull. Amer. Math. Soc., v. 31, 1925, pp. 145-156. 